\title{
Transport Spectroscopy of an Impurity Spin in a Carbon Nanotube Double Quantum Dot
}

\author{
S. J. Chorley, ${ }^{1}$ G. Giavaras,${ }^{2}$ J. Wabnig, ${ }^{2}$ G. A. C. Jones, ${ }^{1}$ C. G. Smith, ${ }^{1}$ G. A. D. Briggs,${ }^{2}$ and M. R. Buitelaar ${ }^{1} *$ \\ ${ }^{1}$ Cavendish Laboratory, University of Cambridge, Cambridge CB3 OHE, United Kingdom \\ ${ }^{2}$ Department of Materials, University of Oxford, Oxford OXI 3PH, United Kingdom
}

(Received 10 September 2010; published 16 May 2011)

\begin{abstract}
We make use of spin selection rules to investigate the electron spin system of a carbon nanotube double quantum dot. Measurements of the electron transport as a function of the magnetic field and energy detuning between the quantum dots reveal an intricate pattern of the spin state evolution. We demonstrate that the complete set of measurements can be understood by taking into account the interplay between spin-orbit interaction and a single impurity spin coupled to the double dot. The detection and tunability of this coupling are important for quantum manipulation in carbon nanotubes.
\end{abstract}

DOI: 10.1103/PhysRevLett.106.206801

Spin qubits defined in carbon nanotube quantum dots are of considerable interest for encoding and manipulating quantum information. Because of the absence of hyperfine interaction in the dominant ${ }^{12} \mathrm{C}$ isotope, spin coherence times are expected to be exceptionally long, while the presence of spin-orbit interaction [1-4] may allow for electrical or even optical [5] control of the spin states. However, before carbon nanotubes can find applications in quantum information processing schemes, we need to understand and control the coupling between individual electron spins and the interaction of the electron spins with their environment.

A powerful method to probe the spin system of quantum dots is to measure the electron transport in a double quantum dot device in the spin blockade regime [6]. In this transport regime, the tunneling of an electron between the quantum dots is forbidden by spin selection rules; hence, the current is suppressed. However, the spin blockade can be lifted by the interaction of the electron spins with their environment, and a measurement of the (leakage) current thus directly probes these interactions. The main spin relaxation and decoherence mechanisms in carbon nanotubes that have been considered so far are spin-orbit coupling and hyperfine interaction in ${ }^{12} \mathrm{C}$-enriched nanotubes $[7,8]$. However, a further important consideration in any realistic nanotube device is the presence of bends, impurities, or defects and their coupling to the electron spins. In this work we make use of the excellent spin sensitivity in the spin blockade regime to investigate the spin system coupling of a carbon nanotube double quantum dot and spin impurities in its environment. In a series of detailed measurements, we show how the interplay of a single impurity spin and the spin-orbit interaction affects the spin states of the nanotube double quantum dot and demonstrate that the coupling to spin impurities can be tuned by gate electrodes.

The device that we consider is a single-walled carbon nanotube grown with natural isotope ratios contacted by $\mathrm{Au}$ electrodes. Side and top barrier gates are used to define

PACS numbers: 73.63.Kv, 71.70.Ej, 73.23.Hk, 73.63.Fg

and control the double quantum dot; see Fig. 1(a). A typical charge stability diagram of the device is shown in Figs. 1(c) and 1(d) in which the ordered pairs $(n, m)$ indicate the effective electron occupancies of the many-electron double quantum dot. In the presence of a source-drain bias voltage $V_{\mathrm{sd}}=-1 \mathrm{mV}$, a honeycomb structure, characteristic of a double quantum dot [9], is clearly visible. The large-small-large-small alternation of

(a)

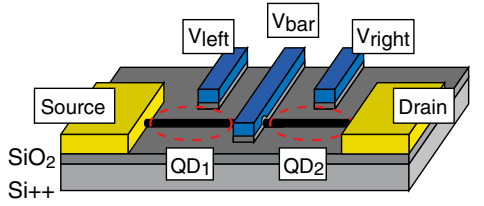

(b)

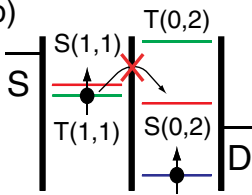

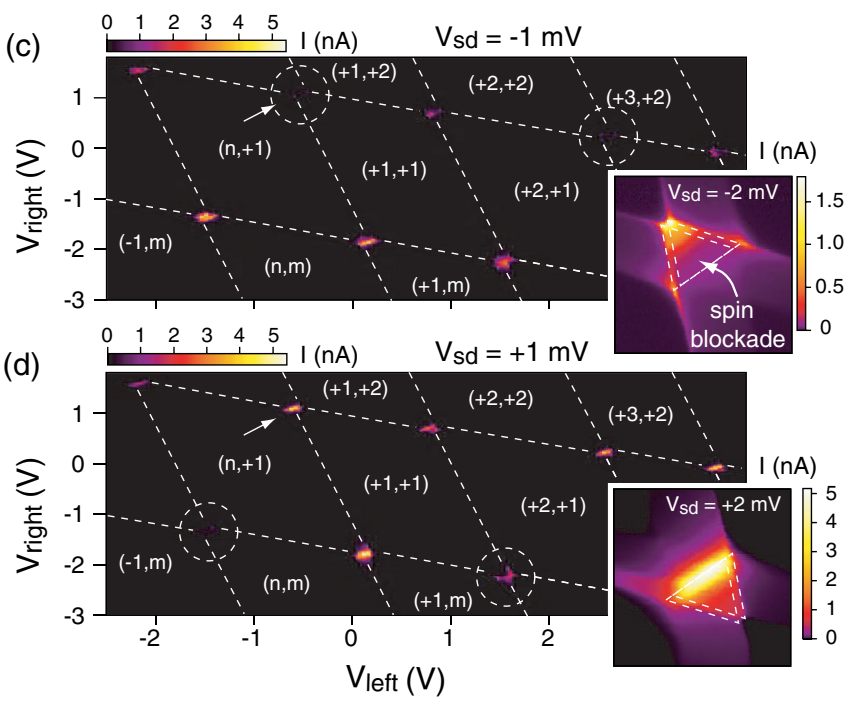

FIG. 1 (color online). (a) Schematic of the carbon nanotube double quantum dot device. (b) Energy level diagram of the double quantum dot in the spin blockade regime. (c),(d) Charge stability diagrams for $V_{\text {sd }}=-1 \mathrm{mV}$ and $V_{\text {sd }}=+1 \mathrm{mV}$ measured at temperature $T \sim 60 \mathrm{mK}$. The circles indicate the regions of spin blockade. Insets: Detailed measurements of the bias triangles at the $(0,1)$ to $(1,2)$ charge transition measured at $\pm 2 \mathrm{mV}$ bias. Note the difference in the current scales. 
the addition energy in the honeycomb pattern indicates that the electron states are spin degenerate but that the orbital degeneracy of the nanotube [10] has been broken.

Of particular interest is the observation of Pauli spin blockade [6,11], of which a characteristic feature is the strong bias dependence of the current for every other added electron as seen in the top and bottom rows of Figs. 1(c) and 1(d), respectively. This is further illustrated by the detailed measurements in the insets in Figs. 1(c) and 1(d) that correspond to the gate region, indicated by the arrows, in which the excess number of electrons changes from $(0,1)$ to $(1,2)$. For negative bias voltage the current is strongly suppressed, while a large current is observed at the base of the bias triangles when the bias is positive.

These measurements can be understood by considering that, for negative bias, a flow of electrons from the left to the right quantum dot necessarily involves a transition from the $(1,1)$ to the $(0,2)$ charge state. Since the $(0,2)$ ground state has to be a singlet by virtue of the Pauli exclusion principle, the $(1,1) \rightarrow(0,2)$ transition is forbidden by spin selection rules when the electrons on the double dot form a $T(1,1)$ triplet state; see also the schematics in Fig. 1(b). When the bias is positive, the $S(0,2) \rightarrow$ $S(1,1)$ transition involves singlet states only, electrons can freely move from the right to the left quantum dot, and no current suppression is observed.

In the following, we make use of spin selection rules to directly probe the spin system of the double quantum dot in detail. Our main results are illustrated in Fig. 2. The top row shows the stability diagrams of spin-blockaded bias triangles for five different barrier potentials. The bottom row shows the current through the double dot as a function of magnetic field $B$ perpendicular to the nanotube axis and detuning $\epsilon$ for three fixed barrier potentials $V_{\mathrm{bar}}=0,10$, and $15 \mathrm{mV}$, corresponding to three of the stability diagrams in Fig. 2(a). Note that in Fig. 2(b) we plot the normalized current to accentuate the evolution of the spin states by effectively subtracting the background current. The complete measurement set, for all measured barrier voltages, and examples of measurements at other bias triangles are shown in Ref. [12].

As evident from the measurements presented in Fig. 2(b), the observed evolution of the spin states can be very rich and, as discussed below, deviates considerably from naive expectations based on the simple even-odd spin filling pattern observed in Fig. 1. We start with a description of the current dependence at $V_{\mathrm{bar}}=0 \mathrm{mV}$, corresponding to the leftmost stability diagram in Fig. 2(a). In the Pauli blockade regime, only the $(1,1)$ triplet states are occupied and no current can pass through the device. However, when the magnetic field is nonzero, the degenerate triplet splits, and for a given magnetic field and detuning the triplet energies equal the hybridized singlet energies; see the schematics in Fig. 3(a). In the presence of spin relaxation such as due to a spin-orbit mediated electron-phonon interaction [4], the spin-flip rate is much higher at these points and provides an efficient escape route from the blockaded triplet state. Thus measuring the current as a
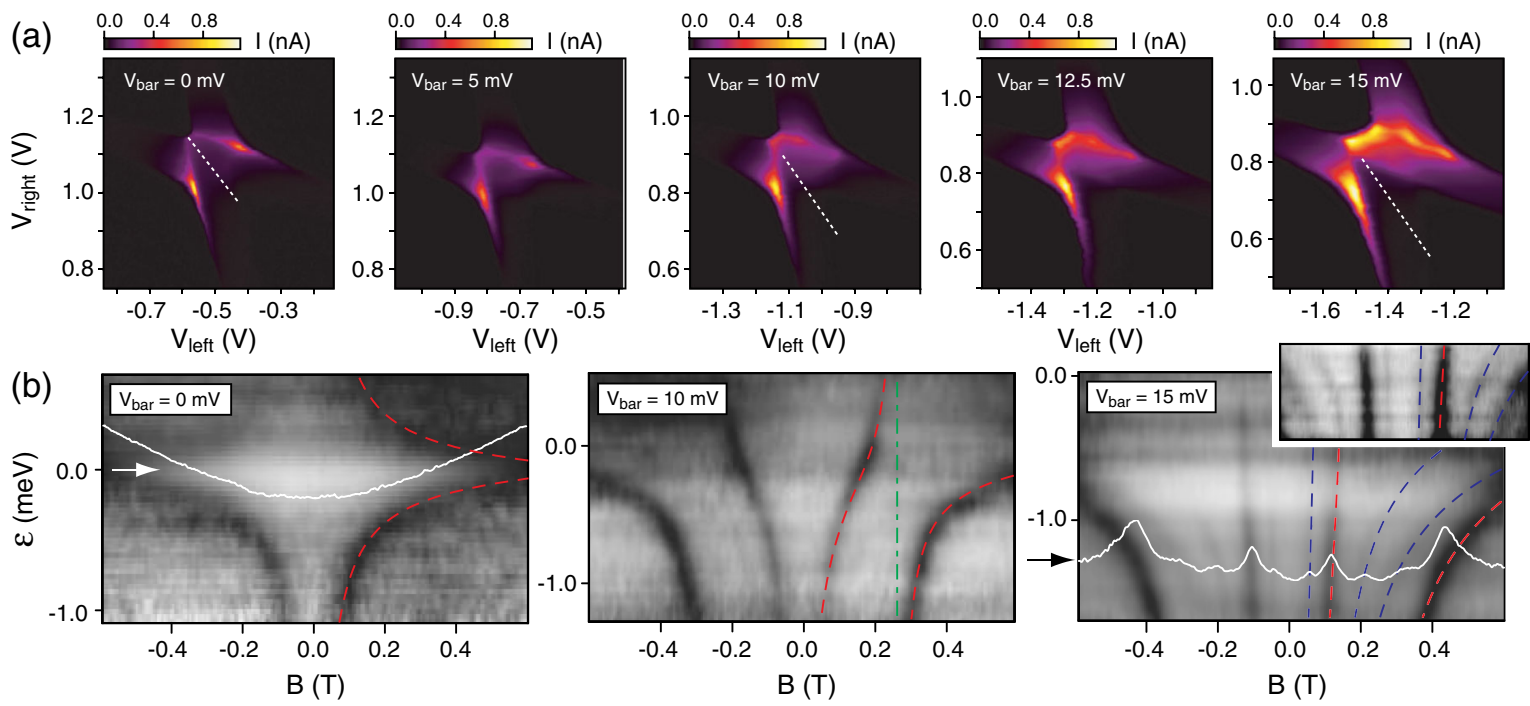

FIG. 2 (color online). (a) Bias triangles at the $(0,1)$ to $(1,2)$ charge transition for $V_{\text {sd }}=-1 \mathrm{mV}$ and $B=0 \mathrm{~T}$ for five different barrier gate voltages. (b) Normalized current as a function of the detuning and magnetic field for $V_{\text {bar }}=0,10$, and $15 \mathrm{mV}$. The detuning axes follow the dashed white lines in (a) for the three respective barrier gate voltages. In the leftmost panel, two sets of curves of high current are visible. In the middle panel, an additional set of curves appears close to zero magnetic field. The rightmost panel shows a series of additional faintly visible current peaks as illustrated by the line trace and the inset, which shows part of the measurement $(|B| \leq 0.37 \mathrm{~T})$ with enhanced contrast. The evolution with the detuning and magnetic field of the features closely follows that predicted by the model (dashed curves) as described in the main text. The free fitting parameters are the interdot tunnel coupling and the strength of the Heisenberg interaction between the double dot and a spin-1/2 impurity. 


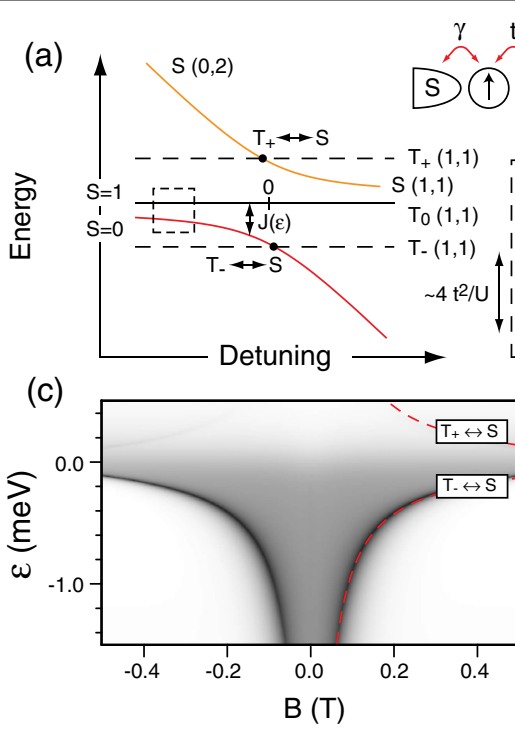

$\stackrel{\gamma}{\sim} \stackrel{\gamma}{\sim} \stackrel{\gamma}{\sim}$

S) (1) (1)

I $\bar{B}=\overline{0}-\bar{B}>0-\overline{m_{S}}$

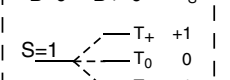

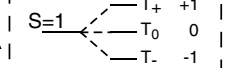

$\mathrm{S}=0$
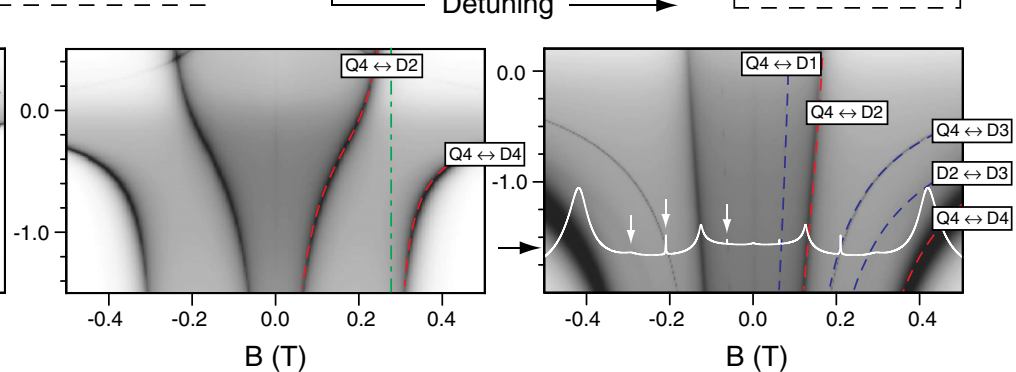

FIG. 3 (color online). (a) Energy of the relevant two-electron states of a double quantum dot as a function of the detuning. The black line represents the $(1,1)$ triplet states $(S=1)$, while the red and orange curves represent mixtures of a $(0,2)$ singlet state with a $(1,1)$ singlet state $(S=0)$. For nonzero magnetic field, the triplet state energies split. (b) Energy of the relevant two-electron states of a double quantum dot including a spin-1/2 impurity as a function of detuning. The impurity spin couples via an isotropic Heisenberg interaction with a coupling strength $J_{\text {imp }}$ to one of the dot spins; see the inset. The black line represents a $S=3 / 2$ quartet. The remaining curves are $S=1 / 2$ doublets. Spin selection rules allow transitions with $\Delta m_{s}= \pm 1: Q 3 \leftrightarrow D 1, Q 3 \leftrightarrow D 3, Q 4 \leftrightarrow D 2$, $Q 4 \leftrightarrow D 4$, and $D 2 \leftrightarrow D 3$, while other, forbidden, transitions are $Q 4 \leftrightarrow D 1$ and $Q 4 \leftrightarrow D 3$. (c) Calculated current as a function of the magnetic field and detuning. Left: For no impurity $\operatorname{spin}\left(J_{\text {imp }}=0\right)$ and $t=70 \mu \mathrm{eV}$. Middle: For an impurity spin with $J_{\text {imp }}=8 \mu \mathrm{eV}$ and $t=87 \mu \mathrm{eV}$, no spin-flip tunneling. Right: For impurity spin with $J_{\text {imp }}=6 \mu \mathrm{eV}$ and $t=170 \mu \mathrm{eV}$, in the presence of spin-flip tunneling with $t_{s}=7.5 \mu \mathrm{eV}$. Note that the origin of the curve $D 2 \leftrightarrow D 3$ in the rightmost plot is the large interdot tunnel coupling and not the spin-orbit interaction.

function of the detuning and magnetic field maps out the exact energy dependence of the singlet energies and, therefore, the singlet-triplet exchange energy $J(\epsilon)$, as seen in the leftmost funnel-shaped pattern in Fig. 2(b). The measurements also demonstrate our ability to electrically tune $J(\epsilon)$ by varying the detuning.

We will now turn to the remaining (rightmost) two panels of Fig. 2(b). When the barrier voltage is set to $V_{\text {bar }}=10 \mathrm{mV}$, a completely different pattern appears in which two sets of curves that approach a common asymptote at $B \sim 0.26 \mathrm{~T}$ are seen; see the dashed-dotted line. Intriguingly, when the barrier voltage is increased further to $V_{\mathrm{bar}}=15 \mathrm{mV}$, the two sets of curves are accompanied by three further, weaker, sets of curves [see the rightmost panel in Fig. 2(b)]. The very different evolution of the spin states in each panel-not seen previously in any double quantum dot system-makes it clear that a detailed understanding of the spin system of the nanotube is required.

To explain our observations, we propose a model where additionally to the electrons on the double dot a third spin, the impurity spin, is present [12]. As justified below, we assume it to be a spin- $1 / 2$ impurity that couples to the spin of the electron on the left quantum dot via an isotropic Heisenberg interaction with a coupling strength $J_{\text {imp }}$; see Fig. 3(b). Experimentally, the strength of the interaction depends strongly on the applied gate bias. When $J_{\text {imp }}=0$, we obtain the conventional spin funnel pattern which provides an excellent fit to the data in the leftmost panel of Fig. 2(b), yielding a tunnel coupling $t=70 \mu \mathrm{eV}$, consistent with an independent estimate of $t$ from the stability diagram in Fig. 2(a). When $J_{\text {imp }} \neq 0$, the relevant states of the combined quantum dot and impurity system can be characterized by their total spin: a fourfold degenerate spin-3/2 state and two doubly degenerate spin- $1 / 2$ states, as shown in Fig. 3(b). The $S=3 / 2$ states cannot mix with the $S=1 / 2(0,2)$ state since tunneling conserves spin and therefore block the current, while all the spin- $1 / 2$ states can take part in transport through the device, having a $(0,2)$ component. The multiplets split in a magnetic field with the energy of states with higher magnetic spin quantum numbers $m_{S}$ passing the energy of states with lower magnetic spin quantum numbers as indicated in Fig. 3(b). In the presence of spin relaxation, transitions involving one spin flip are allowed, corresponding to $\Delta m_{s}= \pm 1$. Since the lowest lying state in the $S=3 / 2$ quartet $(Q 4)$ has the highest occupation probability at finite magnetic fields, we therefore expect to see only two strong curves, corresponding to transitions from state $Q 4$ to $D 2$ and $D 4$, tracing out the shape of the $S=1 / 2$ levels. This is indeed the case as observed in the data [see Fig. 2(b)], which is in excellent agreement with the calculated current as shown in Fig. 3(c). 
The model also allows us to investigate the effects of spin-orbit interaction on the electron transitions. A first indication of the presence of spin-orbit coupling is the zero-field dip in the spin blockade leakage current around $\epsilon=0$ as seen in the leftmost plot in Fig. 2(b). This feature has previously been observed in carbon nanotube double quantum dots and has been tentatively attributed to spinorbit interaction $[7,8,13]$. These results were reproduced in recent theoretical work in which the spin-orbit interaction was shown to introduce non-spin-conserving tunneling between the two quantum dots [14]. In our model, this is characterized by a spin-flip tunneling amplitude $t_{s}$. Since in the presence of a spin-orbit interaction $m_{s}$ is no longer a good quantum number, spin selection rules can be violated, resulting in additional transitions and therefore extra curves in the current plots. These curves are indeed observed in the data, as most clearly seen in the rightmost plot in Fig. 2(b). The position and evolution of the additional three curves are in excellent agreement with those predicted by the model calculation as illustrated by the dashed curves in Fig. 2(b) and the corresponding calculated current in Fig. 3(c).

Spin-orbit interaction also affects the shape of the curves. In the absence of a spin impurity, the spin-flip tunneling induces coherent transitions between $T_{+/-}(1,1)$ and $S(0,2)$ resulting in an avoided crossing between the triplet and singlet states, the size of which depends on the $(0,2)$ component of the singlet. When an impurity is present, this results in a relatively narrow inner curve and a wide outermost curve which increases in width as $\epsilon \rightarrow 0$. A comparison with the data gives fairly narrow bounds for the spin-flip tunneling and yields $t_{s}=$ $7.5 \mu \mathrm{eV}$; see Ref. [12]. Using the estimate from Ref. [14] and an orbital energy $E_{\text {orb }} \sim 2 \mathrm{meV}$, we can deduce the spin-orbit interaction energy as $E_{\mathrm{SO}}=$ $E_{\text {orb }} t_{s} / t \approx 0.1 \mathrm{meV}$. We note that this value is similar to previous estimates $[1,8]$ which is of interest as the strength of spin-orbit interaction is not a priori clear for a carbon nanotube with mixed orbital states and in the manyelectron limit as investigated here.

The above analysis demonstrates that we are able to detect the presence of a single impurity spin coupled to the carbon nanotube double quantum dot and determine its spin quantum number. An important question that remains is the nature of the impurity. Possibilities are the presence of a single atom absorbed on the nanotube wall [15], a charge trap in the gate oxide, or a defect such as a vacancy or dopant in the carbon lattice [16,17].

From a practical perspective, we note that our work and recent results by other groups $[18,19]$ indicate that spin impurities are relatively common in carbon nanotube devices. The observation here of a single impurity spin would be consistent with estimates for high-quality carbon nanotubes of one defect per $4 \mu \mathrm{m}$ on average [20], which implies that a typical device of length $L \sim 1 \mu \mathrm{m}$ will contain either zero or one impurity. Whereas many individual nanotube devices will therefore be without a single impurity spin, requirements on the nanotube quality will become more stringent when many quantum dots are coupled in a large-scale quantum circuit. On the other hand, our experiments also show that the coupling to an impurity spin can be precisely controlled with gate electrodes which suggests the possibility of storing quantum information into the localized (impurity) spin and using the carbon nanotube for spin state readout [21,22]. The ability to control these interactions will be instrumental in developing carbon materials for quantum information processing.

We thank David Cobden and Jiang Wei for the carbon nanotube growth and Brendon Lovett for discussions. This work was supported by QIPIRC, the Wenner-Gren Foundation (J. W.), and the Royal Society (M. R. B.).

*mrb51@cam.ac.uk

[1] F. Kuemmeth, S. Ilani, D. C. Ralph, and P. L. McEuen, Nature (London) 452, 448 (2008).

[2] T. Ando, J. Phys. Soc. Jpn. 69, 1757 (2000).

[3] D. Huertas-Hernando, F. Guinea, and A. Brataas, Phys. Rev. B 74, 155426 (2006).

[4] D. V. Bulaev, B. Trauzettel, and D. Loss, Phys. Rev. B 77, 235301 (2008).

[5] C. Galland and A. Imamoğlu, Phys. Rev. Lett. 101, 157404 (2008).

[6] K. Ono, D. G. Austing, Y. Tokura, and S. Tarucha, Science 297, 1313 (2002).

[7] H. O. H. Churchill et al., Nature Phys. 5, 321 (2009).

[8] H.O.H. Churchill et al., Phys. Rev. Lett. 102, 166802 (2009).

[9] W. G. van der Wiel et al., Rev. Mod. Phys. 75, 1 (2002).

[10] W. J. Liang, M. Bockrath, and H. Park, Phys. Rev. Lett. 88, 126801 (2002).

[11] M. R. Buitelaar et al., Phys. Rev. B 77, 245439 (2008).

[12] See supplemental material at http://link.aps.org/ supplemental/10.1103/PhysRevLett.106.206801 for additional data and details of the model.

[13] Hyperfine mixing would result in a current peak at $B=0$ which is not observed in the data. In our model, we neglect hyperfine contributions to spin relaxation.

[14] J. Danon and Yu. V. Nazarov, Phys. Rev. B 80, 041301(R) (2009).

[15] T. W. Odom, J-L. Huang, C. L. Cheung, and C. M. Lieber, Science 290, 1549 (2000).

[16] Y. Ma, P. O. Lehtinen, A. S. Foster, and R. M. Nieminen, New J. Phys. 6, 68 (2004).

[17] Y-W. Son, M. L. Cohen, and S. G. Louie, Nano Lett. 7, 3518 (2007).

[18] Yu. Bomze et al., Phys. Rev. B 82, 161411 (2010).

[19] A. Eichler, M. Weiss, and C. Schönenberger, arXiv:1008.5103.

[20] Y. Fan, B. R. Goldsmith, and P. G. Collins, Nature Mater. 4, 906 (2005).

[21] J. M. Elzerman et al., Nature (London) 430, 431 (2004).

[22] J. Wabnig and B. W. Lovett, New J. Phys. 11, 043031 (2009). 